\title{
Philosophiques
}

\section{Savoir et croire. Sur Le Pen et autres menus détails}

\section{Richard Poulin}

Volume 16, numéro 2, automne 1989

URI : https://id.erudit.org/iderudit/027085ar

DOI : https://doi.org/10.7202/027085ar

Aller au sommaire du numéro

Éditeur(s)

Société de philosophie du Québec

ISSN

0316-2923 (imprimé)

1492-1391 (numérique)

Découvrir la revue

Citer ce document

Poulin, R. (1989). Savoir et croire. Sur Le Pen et autres menus détails.

Philosophiques, 16(2), 350-371. https://doi.org/10.7202/027085ar d'utilisation que vous pouvez consulter en ligne.

https://apropos.erudit.org/fr/usagers/politique-dutilisation/ 


\title{
SAVOIR ET CROIRE. SUR LE PEN ET AUTRES MENUS DÉTAILS
}

\author{
par Richard Poulin
}

En 1942, exilé au Brésil, Stefan Zweig avale du poison et meurt. Ayant longtemps scruté la catastrophe et le flor obscur qui emportait son monde d'hier - sa culture, ses ami-e-s, ses livres, son pays -, il ne discernait pas alors d'autre moyen d'échapper à la "main du destin ». Dans ses mémoires ${ }^{1}$, achevés peu avant son suicide, il s'interroge sur la stupéfiante cécité manifestée par ses contemporains - allemands, avant tout - face à la montée du nazisme. Les monarchistes, note-t-il avec une ironie trempée dans l'encre la plus noire, étaient convaincus que l'accession de Hitler au pouvoir ne pouvait que constituer un prélude au retour triomphal de l'Empereur ; les nationaux-allemands, c'est-à-dire les nationalistes conservateurs, ne doutaient par un instant que Hugenberg, leur ténor, ne ferait qu'une bouchée du caporal autrichien ; l'industrie lourde se réjouissait de la montée en puissance d'un homme à poigne capable de la délivrer de sa crainte atavique du bolchévisme ; le petit commerçant se voyait déjà débarrassé de la concurrence des grands magasins ; la caste militaire jubilait à entendre les diatribes antipacifistes du Führer ; les sociaux-démocrates eux-mêmes trouvaient plus qu'une consolation à l'idée que, du moins, leur redoutable adversaire saurait mettre les communistes au pas... À ce palmarès de l'aveuglement établi par Zweig, manquent ces mêmes communistes staliniens. C'est dommage: eux aussi étaient convaincus qu'après celui du gnome dérisoire et gesticulant viendrait leur tour, car le déluge nazi n'était qu'un symptôme de l'agonie finale du système capitaliste en crise. 
Zweig n'était pas un politique. Son domaine d'élection, c'était «l'âme humaine », la psychologie des profondeurs. Ses mémoires sont ceux d'un témoin horrifié, non pas d'un acteur. Et pourtant, son étonnement désespéré et sa méditation sur l'aveuglement face à l'horreur montante nous frappent par leur actualité.

La très grande force de personnages comme Mussolini, Hitler ou Le Pen ${ }^{2}$ est de faire irruption dans l'arène politique comme des pitres, des bateleurs. Si vulgaires dans leur démagogie, tant excessifs dans leurs gesticulations, inconsistants dans leurs divagations idéologiques - si différents des politiciens traditionnels - qu'on ne les prend pas au sérieux. Des clowns, des Augustes pour faire rire ou se faire peur le temps d'un petit tour de piste. Depuis que Le Pen connaît le succès électoral et médiatique, on ce cesse de répéter en France que c'est Poujade ${ }^{3}$ qui recommence et que tout ceci ne durera que le temps d'une foire ou d'une crise nécessairement destinée à s'effacer au profit de jours meilleurs; on ne cesse de répéter que, pour fâcheuses que soient les proportions prises par la baudruche, il ne faut pas confondre : Le Pen c'est moche; mais rien à voir, Dieu nous garde, avec Hitler, le nazisme, les camps de concentration. Une petite crise d'acné, par la peste brune.

2. Jean-Marie Le Pen, président du Front national est né à la Trinité-sur-Mer en 1928. Sous-lieutenant au premier baraillon étranger de parachutistes en Indochine, il revient avec « la révélation concrète de l'ennemi communiste, de ses méthodes terribles, de sa manière impitoyable de liquider ses adversaires, de sa technique de guerre impitoyable » (Jean-Marie Le Pen, Les Francais d'abord, Paris, Carrère/Laffon, 1984, p. 34). L'humiliation indochinoise et la lutte de libération nationale algérienne le conduisent à militer pour Poujade au sein de l'UCDA, Union de Défense des Commerçants et Artisans, parti politique populiste éphémère. Élu député de la Seine en 1956, il rompr avec Poujade pour se rapprocher du Groupe des Indépendants et Paysans. Secrétaire général du Front des Combattants puis du Front pour l'Algérie française, il part combattre en Algérie. Il s'oppose à la politique du général de Gaulle donnant l'autodétermination à l'Algérie. Il revient à la politique électorale en tant que secrétaire général du Comité Tixier-Vignancour, candidat d'extrême-droite aux présidentielles de 1965. Le Pen se brouille avec ce dernier et, en 1972, après des négociations avec l'organisation Ordre Nouveau, il crée le Front national qui le nomme président.

3. Pierre Poujade paperier de Saint-Céré, préside la création de l'Union de Défense des Commerçants et Artisans en 1953 dont les thèmes sont l'antifiscalisme, le dirigisme étatique, la bureaucratie tentaculaire et l'incapacité des politiciens. Réaction corporative, I'UCDA exprime les sentiments diffus de ceux qui se sentent menacés par les changements économiques. Nationaliste et populiste, I'UCDA obtient en 1956, 2500000 voix mais éclate dès 1958 au moment de l'arrivée au pouvoir du général de Gaulle. L'UCDA constiruait une sorte de front du refus de la modernisation rapide. 


\section{LA BANALISATION DE L'HORREUR}

Ce qui nous intéresse ici en premier lieu, c'est moins la validité de telle ou telle comparaison historique que cet irrépressible besoin de sécurisation qui, nécessairement, nous ramène à $Z$ weig. Cet horrible sang-froid, cette assurance ignoble qui, d'un revers de la main, congédie la rage et le dégoût, la répulsion et la haine. Cette indéfectible propension à détourner le regard de ce qui constitue le monstre en tant que monstre - sa monstruosité - cette réticence à l'appeler par son nom. On s'épivarde contre ses douteux jeux de mots jusqu’à insister pour lever son immunité parlementaire, tout en comprenant son racisme sous prétexte qu'il soulève de vraies questions malgré de fausses réponses ${ }^{4}$. Tout concourt ainsi à banaliser le mal et à accoutumer les gens à vivre dans sa proximité, à cohabiter avec lui, comme on s'habitue à vivre dans les parages d'une usine chimique dont les cheminées déversent jour et nuit des fumées empoisonnées. Tout: un certain discours historien dont les verdicts ( «national-populisme à la française», mais non le fascisme) sont salués par force soupirs de soulagements ${ }^{5}$; la légitimation médiatique fondée sur l'équation : «représentatif » = fréquentable au même titre que "les autres » ${ }^{6}$; une trompeuse ambiance de paix civile (malgré bavures et ratonnades ${ }^{7}$ régulières mais non massives... encore !) où l'on peut voir le monstre prospérer non point au fil de l'émeute, du cataclysme, du progrome ou de la guerre, mais d'élections, de prestations télévisées, de processions sans plaies ni bosses aux pieds de Jeanne D'Arc... Non, décidément, la bête n'est pas bien méchante; elle ne mord guère qu'en paroles et se contente d'engraisser au soleil de la crise!

4. Propos tenus par Laurent Fabius, dirigeant du Parti socialiste, à l'époque premier ministre du gouvernement jusqu'aux élections législatives de 1986 qui verra la droite l'emporter.

5. Voir entre autres les travaux de Pierre MiLZA, notamment Fascisme français, passé et présent, Paris Flammarion, 1987 ainsi que ceux de Ariane Chebel d'APPOLONIA, L'extrême-droite en France, de Maurras à Le Pen, Bruxelles, Éditions complexe, 1988.

6. Alain Rol.t.AT, journaliste du Monde, auteur de Les Hommes de l'extrême-droite, Le Pen, Ortiz et les autres (Paris, Calman-Levy, 1985) et co-auteur avec Edwy Pl.ENEl, de L'Effet Le Pen (Paris, Le Monde-La découverte, 1984) a souligné avec force le rôle des médias et leur responsabilité dans la légitimation du Front national dans une communication intitulée « Le Pen, miroir de la France » au colloque de l'Union démocrarique; Centrale française démocratique des travailleurs (CFDT), tenu à Paris en mars 1988.

7. De raton, injure raciste argotique française, comme bicot, bougnoule. Ratonnade expédition punitive ou brutalités exercées par des Européens contre des NordAfricains. 
Même lorsque le Front national grimpe à plus de $14 \%$, on se rassure : cette force politique est encore bien petite comparée aux partis démocratiques. Et surtout, elle empiète efficacement sur le terrain d'élection des communistes, force autrement « dangereuse »! Mais il faut apprendre d'autres regards que la seule arithmétique électorale, se contraindre à d'autres questions : quels sont l'écho et l'enracinement des idées et représentations du Front national dans la police, dans l'armée - à la lumière de certains « faits divers ${ }^{8}$ ou de certains événements néo-calédoniens ${ }^{9}$, par exemple? Quelle variante possible, quelle dynamique d'avenir éventuelle dessinent les déclarations (et les gestes subséquents) de l'ex-ministre de l'Intérieur, Charles Pasqua ${ }^{10}$, sur les «valeurs communes» entre la droite et l'extrême-droite, l'accord «local» entre le patron marseillais de l'Union Démocratique Française (U.D.F.) avec le Front national? Ici, il n'est pas mauvais de se rappeler un peu d'histoire - pour penser l'inconcevable - ; comme le signale Zweig, Hitler bouffa bien tout crus les puissants nationaux allemands, dans l'enthousiasme mutuel. Il n'est plus question d'élections, mais de dynamique, de conjonction de facteurs, de conjoncture possible. Et, toujours dans le fil de Zweig : qui, évaluant d'un œil de

8. Voir à ce propos l'excellente étude de S. DUMONT, J. LoRIEN et K. CRITON, Le système Le Pen, Anvers, Éditions Epo, 1985. Après 1968, le ministère de l'Intérieur multiplie les polices antigauchistes et c'est dans ce cadre que nombre d'éléments d'extrême-droite se sont introduits dans différents services de police et/ou ont été utilisés par eux. Voir aussi S. Ferrand et G. LeCaval.Ier, $A u x$ ordres du $S A C$, Paris, éditions Albin Michel, 1985 ainsi que J.-M. PONTAut, Les secrets des écoutes téléphoniques, Paris, Artaud, 1976. Depuis la percée électorale du Front national, les policiers d'extrême-droite ont été impliqués dans de nombreuses " bavures ", notamment des assassinats d'Arabes et de Noirs, comme s'ils bénéficiaient d'une sorte dimpunité.

9. L'imbrication entre les forces de «l'ordre " et l'extrême-droite est telle en NouvelleCalédonie que rares sont les événements qui n'échappent pas au contrôle du gouvernement métropolitain qui, après moultes hésitations, arrive toujours à les couvrir. Voir à propos les informations régulièrement publiées dans le magazine parisien Article 31, magazine voué à la défense des droits de la personne et contre le racisme ainsi que les livres de Claude Gabriel et Vincent Kermel, Nouvelle-Calédonie, la révolte Kanake, et Nouvelle-Calédonie, les sentiers de l'espoir, Montreuil, éditions La Brèche, 1985 et 1988.

10. Charles Pasqua, ministre de l'Intérieur sous le gouvernement de Jacques Chirac (1986-1988) représentait la tendance de la droite qui espérait réussir à s'allier durablement l'extrême-droite et mêrne, peut-être, gouverner avec elle. Pour ce faire, it fir adopter une loi très dure contre les immigrés, les déportant par charters complets, et a tenté d'imposer le Code de nationalité qui a été retiré après les grandes manifestations étudiantes de décembre 1986. 
profane ou de spécialiste l'«effet Le Pen», n'est pas, un jour ou l'autre, tombé dans le piège du «à quelque chose malheur est bon »? Qui n'a pas retrouvé dans le propos du démagogue l'écho plus ou moins assourdi de son propre credo? N'oublions jamais ce très large consensus larvé autour de l'utilité de l'extrême-droite: pour les uns, il était heureux qu'elle fût là pour jeter la zizanie parmi les droites - et ils lui en ont largement fourni les moyens ; pour les autres, il fallait bien admettre que le bateleur vulgaire «posait les vraies questions»; pour d'autres encore, il convenait de reconnaître les évidents mérites de sa foi libérale, au sens reaganien du terme; pour d'autres encore et encore, il fallait bien saluer en lui le patriote soucieux de l'identité française et de l'intégrité nationale; sans oublier ceux qui, naguère «très rouges » et "pas racistes pour un sou», se réjouissent de voir le loup parachever l'œuvre salutaire de liquidation du Parti communiste français dans ces banlieues désespérées par le chômage et la " galère " "'... Qui, face à la bête, n'a jamais cédé à la joie maligne de la voir mordre l'adversaire au talon. Petit calcul intéressé, politique politicienne «réaliste», petit coup de pouce à la banalisation de l'horreur.

Que l'on nous entende bien : il ne s'agit pas ici simplement de stigmatiser telle action raciste du Parti communiste français ${ }^{12}$, telle mancuvre retorse du Parti socialiste ou telle évidente manifestation de la contamination croissante de la droite dite classique par les miasmes des égouts. Confrontée aux catastrophes imminentes (1914, 1933 en Allemagne, 1939-1940 en France, 1936 en Espagne, etc.), la social-démocratie n'admet de leçons de cécité de personne :

11. Terme argotique français qui réfère à une situation ou une condition excessivement pénible

12. Indiquons ici que le Parti communiste français reprend à son compte depuis des décennies une propagande typiquement nationaliste à grands coups de "Fabriquons français » et d' « Achetons français ». En 1981, lors des élections, entre les deux tours, le dérapage face aux travailleurs étrangers se produit à coups de bulldozers dans la municipalité communiste de Vitry. En plein hiver, on chassait des travailleurs maliens en détruisant leur malheureux et miséreux foyer. Au même moment, les communistes de Montigny désignaient à grand renfort de dénonciations et délations à la vindicte publique une pauvre famille de travailleurs marocains accusée à tort de vendre de la drogue à la jeunesse française. Voir à ce propos, Philippe RoBrieux, (Histoire intérieure du Parti communiste, 1972-1982, tome 3, Paris, Fayard, 1982, troisième partie, notamment les pages 433 à 441 . 
regroupée autour du drapeau national, elle entonne ses cantiques patriotiques et ferme les yeux dans l'espoir que la bête se contentera de dévorer les rouges sans piétiner les roses. Les communistes staliniens en arrivent même à préférer le brun au rose, à faire de la surenchère dans le brun, à assassiner (Espagne républicaine, résistance française, camps de concentration nazis) plus rouges qu'eux ${ }^{13}$. Quant à la droite "classique», elle ne constitue en rien, dans la tradition française, une entrave sérieuse à l'extrême-droite activiste, une sorte d'essence démocratique, opposée à une autre fascisante, fasciste, totalitaire. Le gaullisme, sa guerre contre l'OAS ${ }^{14}$ et son habile gestion du patrimoine de la Résistance ont, de ce point de vue, brouillé les cartes malgré le coup d'État bonapartiste de $1958^{15}$, : mais le paysage de la droite française a une histoire plus ancienne : le front antisémite anti-dreyfusard, les ligues des années trente, la lutte contre le Front populaire et le soutien à l'Espagne franquiste, la collaboration pendant l'occupation nazie, l'anticommunisme de guerre froide et les crispations néocolonialistes ${ }^{16} \ldots$ Sur toutes ces scènes, la droite que certains appellent «civilisée » et celle dont ils admettent qu'elle ne l'est guère s'interpénètrent davantage qu'elles ne se distinguent comme essences, comme blocs ${ }^{17}$. Rappelons que " l'académie » de la Nouvelle droite qu'est le Club de l'Horloge ${ }^{18}$ n'est pas davantage

13. Un ancien résistant communiste a écrit un livre accablant sur ces événements : René DAZY, Fusillez ces chiens enragés!..., Paris, éditions Olivier Orban, 1981

14. Organisation Armée Secrète. Contre l'indépendance de l'Algérie, après l'avortement d'un coup d'État déclenché le 22 avril 1961 par les militaires en poste à Alger, l'OAS nait dans la clandestinité pour mener un combat d'arrière-garde contre le Front de libération national algérien. L'OAS étendra ses activités en métropole et s'assurera le sourien des militants d'extrême-droite, dont plusieurs deviendront des dirigeants du Front national, notamment Pascal Arrighi.

15. Rappelons que le coup d'État gaulliste de 1958, acte fondateur de la Ve République, érait combattu jusqu'en 1981 par la gauche socialiste et communiste parce que la Constiturion concentrait trop de pouvoirs discrétionnaires à la présidence de la République. Acte anti-démocratique, cette Constitution devait être fortement amendée et l'Union de la Gauche visait un changement de régime en faveur d'une VIe République ce qui a été mis aux oubliettes depuis.

16. Voir mon article, “Étreintes coloniales sur les Cailloux », Critiques socialistes, automne $1988, n^{\circ} 4$, pp. 115-126.

17. Voir à ce propos les ouvrages de A. RoH.aT, op. citi., note 6 .

18. Créé en 1974, le Club de l'Horloge, lié clairement jusqu'en 1979 à l'extrême-droite, se veut un lieu de réflexion dénonçant l'utopie égalitaire, se référant à la sociobiologie pour souligner les différences entre les peuples et les cultures et tentant d'élaborer « un projet politique de stratégie haute de contournement du socialisme sur ses positions ». Pour vaincre le socialisme «qui sape les capacités de résistance matérielles et spirituelles 
lépéniste que chiraquien ${ }^{19}$. On peut aussi se demander ce qui, tout bien pesé, concernant l'avenir de la Nouvelle-Calédonie, distingue les rêves et cauchemars de la droite de ceux de l'extrême-droite ${ }^{20}$.

\section{Y CROIRE OU NE PAS Y CROIRE?}

Mais là n'est pas l'essentiel. L'essentiel est qu'au fond, personne ne "croie» vraiment que le Front national pourra prendre le pouvoir, c'est-à-dire à son aptitude à produire la catastrophe. Le Front national irrite, répugne, inspire honte (d'être français) et parfois amusement, mais en réalité, il ne fait pas vraiment peur, sauf aux Arabes, aux Juifs, aux Antillais et aux Africains. On n'y croit pas - au sens où Zweig s'étonne que l'on n'y ait pas cru. Au fond, face au $14 \%$ en faveur de Le Pen aux élections présidentielles de 88 (10\% aux élections législatives de la même année), tout le monde, et pas seulement les démocrates perclus d'illusions démocratiques, croit spontanément à la démocratie (tradition occidentale/ judéo-chrétienne, etc.), à la paix civile, à l'inébranlabilité des institutions qui en garantissent la pérennité. Ja mais le discours sur la démocratie n'a été aussi imposant sur l'idée elle-même, non sur la chose, ses fonctions, ses limites... On veut croire bien davantage à la démocratie qu'à sa fin possible.

Face au caraclysme possible, on préfère, en règle générale, croire en l'éternité des choses que savoir. En 1933, les démocrates

face au danger extérieur », le Club de l'Horloge entend défendre des valeurs telles que "liberté, sécurité, propriéré et résistance à l'oppression ". Il s'oppose à l'État-providence, au bureaucratisme et au fiscalisme. Voir à ce propos l'ouvrage du Club de l'Horloge, d'où viennent les citations, Échecs et injustices du socialisme suivi du Projet républicain pourl'opposition, Paris, Albin Michel, 1982, pp. 153, 152 et 214. Depuis 1979, le Club de l'Horloge regroupe en son sein des penseurs de la droite dite classique et de l'extrême-droite, créant une sorte de carrefour de réflexion. Ainsi ce Club est directement lié à l'Union pour la Démocratie Française, au Rassemblement pour la République, au Centre National des Indépendants et Paysans, au Front national et au Groupement de Recherche et d'Études pour la Civilisation Européenne (GRECE), dirigé par Alain de Benoist, sorte de Club de réflexion pour les néo-nazis et l'extrême-droite plus " modérée ». Voir S. DUMONT, J. LORIEU, K. CRITON, op. cit., notamment l'organigramme publié aux pages 306-307.

19. Jacques Chirac, maire de Paris, leader du Rassemblement pour la République, ancien premier ministre sous les septennats de Giscard d'Estaing et de François Mitterrand, notamment dans ce dernier cas de 1986 à 1988.

20. Voir à nouveau des deux ouvrages op. cit., de Claude Gabriel et Vincent Kermel, notamment celui publié en 1988. 
et les Juifs d'Allemagne continuaient à croire que les institutions de la République de Weimar et les traditions de la grande culture allemande pèseraient davantage que les billevesées et les scénarios d'apocalypse de Hitler. Et pourtant, il leur était bien difficile de ne pas savoir les intentions du Führer. En pleine extermination des Juifs d'Europe, le président Roosevelt et les dirigeants de la communauté juive américaine, submergés d'informations alarmantes concernant les camps et les chambres à gaz n'y croyaient pas : ce génocide en marche n'entrait pas dans leurs catégories et représentations : un peuple civilisé, signataire du traité de Genève, ne peut pas faire ça ${ }^{21}$. Et puis, à un niveau plus prosaïque, cela dérangeait leurs plans et leur routine, de le savoir et d'y croire.

\section{L'ILLUSION RÉTROSPECTIVE}

Dans un contexte où les enjeux de cette dialectique du savoir et du croire ne se comparent guère, évidemment, nous assistons néanmoins au retour de ce même goût de ne pas savoir. Les médias d'information aussi bien que les ténors de la social-démocratie sont passés maîtres dans cet art spontané du wishful thinking face au Front national: qu'il trébuche lors d'élections locales, qu'un ancien lieutenant du bateleur vienne cracher dans sa soupe, que sa fraction parlementaire se lézarde, qu'une opportune réforme du mode scrutin vienne réduire cette dernière à la portion congrue, et c'est déjà l'hallali: on vous l'avait bien dit, cela ne pouvait pas durer. Ou bien alors, c'est l'illusion rétrospective, sur fond de minables calculs électoralistes, qui vient enfoncer le clou: le fascisme, c'est les foules déchaînées et fanatisées dans la rue (ce qui n'était sans doute pas le cas des 100,000 manifestants du $1^{\text {er }}$ mai 88 en faveur de Le Pen et de Jeanne d'Arc!), les livres qui brûlent, les Juifs exterminés, la guerre... Non rien de tout cela avec Le Pen. Donc, ses quatre millions d'électeurs (auxquels on tend une main fraternelle et intéressée) ne sauraient en aucun cas être vitupérés comme «fascistes », « antisémites » ou « violents» racistes. Même Anne Tristan, avant la réédition de son livre, $A u$ Front ${ }^{22}$, et sa

21. Voir à ce propos: Walter LAgneur, Le Terrifiant secret, Paris, Gallimard, 1981 et David S. WYMAN, L'Abandon des Juifs, Les Américains et la solution finale, Paris, Flammarion, 1987

22. Anne Tristan, Au Front, Paris, Gallimard, 1987, réédition dans la collection de poche, 1988. 
postface cinglante contre l'extrême-droite et sa banalisation par le Parti socialiste et la droite, en relatant son expérience au Front national de Marseille, insistait sur la "gentillesse de l'ennemi»: bref, le porteur d'eau du Front est un Français comme tous les autres, dont on a abîmé la vie et qui en bave dans ses quartiers de haute insécurité...

Ces commis en assurance et rassurance n'oublient qu'une chose : la population désorientée, désespérée, enragée qui a porté Hitler au pouvoir était, elle aussi, "gentille» : elle ne rêvait pas de chambres à gaz, n'avait rien de particulier contre Einstein, Thomas Mann ou les Russes. Elle n'était qu'une masse déboussolée par le chômage et la misère (et ce qu'en dit Tristan pour les quartiers nord de Marseille est éclairant), un agrégat de rancœurs et de colères prêt à se coaguler et se «transcender » dans des délires et aventures imaginaires. Ce à quoi nous assistons en France aujourd'hui (et plus globalement en Europe) y ressemble furieusement : cette conjonction dans un mouvement impétueux de rages aveugles et d'intérêts disparates, cette mise en discours, en idéologie, d'aigreurs, d'angoisses dispersées, ce miracle des solutions aussi limpides qu'imaginaires: trois millions de chômeurs, trois millions d'immigrés...

Il n'y a pas de raisons particulières de trouver à la population lépéniste de 1989 meilleure figure qu'à celle nazie de 1930 . Ce que disait Trotsky ${ }^{23}$ de la seconde - une humanité «en miettes», atomisée et enragée - s'applique à la première.

Ces évidences, les intellectuels démocrates les oublient parce qu'ils ne savent que penser l'éternité du moment présent : lorsque brille le soleil de la prospérité, ils transmuent en «valeurs", en éthique sociale, les dividendes de la bonne fortune du système; lorsque le ciel s'assombrit, ils ouvrent le parapluie philosophique de l'individualisme, de la nécessaire concurrence, de l'autorité et de la démocratie comme idée, celle des virtualités, pour les individualités, de l'éphémère et du vide. Leur seul credo est au fond celui du bienfondé du moment présent. C'est pourquoi ils n'arrivent pas à penser la barbarie en gestation et les catastrophes possibles. Cette perception de l'histoire, malgré discours et contre-discours sur les

23. Léon TRotsky, Comment vaincre le fascisme, Paris, Buchet-Chastel, 1973. 
nouveaux mouvements sociaux, en arrive à confondre le réel avec la composition de l'Assemblée nationale (plus ou moins de représentants du Front national). Elle n'est pas cette perception des courants souterrains de l'histoire qui permet de concevoir et le meilleur et le pire possibles ce qui, en définitive, détermine l'action. Le Pen est nécessairement inconcevable comme agent de la catastrophe pour qui, tout à ses petites certitudes démocratiques et patriotiques, croit que les consensus encore dominants de la "civilité» française sont éternels. Mais combien de fois, de la Commune de Paris à la guerre d'Algérie les a-t-on vus s'effondrer? Gorbatchev est nécessairement inintelligible pour qui, tout à son credo antitotalitaire, a toujours confondu l'immobilité des tours du Kremlin avec le mouvement de la société soviétique. Défendre la civilisation, la démocratie, ne serait-ce pas avant tout s'opposer à l'asiate russe barbare (le communisme et a fortiori le mouvement ouvrier, «sujet totalitaire de l'histoire ») et donc ne voir en Le Pen qu'un trublion qui d'ores et déjà accepte et intègre le jeu démocratique?

Écartons donc toute illusion rassurante: le phénomène Le Pen n'est ni conjoncturel ni éphémère. Le tremblement de terre a bien eu lieu et il a déstabilisé le champ politique français. Son ressac a atteint presque toute l'Europe. En Norvège, le Parti du progrès, mélange d'ultra-libéralisme et d'antisémirisme, réalisait $12,17 \%$ aux élections municipales de 1987. En juin 1988, les sondages lui donnaient $23 \%$ des voix. En Suisse, les fascistes de Vigilance et d'Action nationale, au Conseil municipal de Lausanne, en 1985 , ont obtenu 16 sièges en ne présentant que 12 candidats ${ }^{24}$. Le nouveau parti, le Parti des automobilistes obtient $10 \%$ des voix. "Il faut brûler les écolos au lance-flammes», clamait leur leader, Michaël Dreher. "Nous sommes un authentique parti populaire... Nous entendons défendre les droits des automobilistes contre le péril vert... La télévision et la radio sont tenues par la gauche. Nous voulons sauvegarder la vie libre et motorisée! »Un programme loufoque... et dangereux. En Italie, le MSI, Movimento

24. En Suisse, le mode d'élection municipal est proportionnel. C'est dire qu'une liste peut recevoir un pourcentage de votes plus élevé que prévu et donc obtenir plus de sièges qu'elle n'a de candidats inscrits. Le parti aura donc à nommer en son sein ses élus au Conseil municipal. 
Sociale Italiano, récolte toujours de $6 \%$ à $7 \%$ des voix. En 1987, il avait trente-cinq députés, seize sénateurs et quatre députés européens. En Autriche, le pays de Waldheim, le Parti libéral a été repris en main il y a quelques années par Joerg Haïder. En 1986, il s'offrait $9,72 \%$ des voix et depuis sa cote ne fait que grimper. Ultralibéraliste, populiste et autoritaire, sans réel problème d'immigration, il lui fallait trouver d'autres thèmes. État musclé et assainissement draconien du secteur public. Anti-parlementarisme, un brin de pangermanisme et surtout un soutien bruyant à Waldheim contre le «complot cosmopolite ». Là où on ne craint pas les Arabes, on peut toujours ressortir les Juifs. Haïder déclarait à l'été 88 : «Si Le Pen est un extrémiste de droite, alors les socialistes sont des marxistes-léninistes ». $5 \%$ à Rotterdam, aux Pays-Bas ; à Malmö en Suède, les dernières municipales ont vu $9 \%$ des électeurs voter pour le Parti de la Suède ; $9 \%$ aussi au Danemark pour la Parti du progrès, parti ouvertement raciste (élections législatives de mai 1988).

«Comment! moi, national-socialiste? J'ai toujours détesté les socialistes!», clame le leader du Front national de Belgique ( $5 \%$ des voix à Bruxelles). À Berlin, les néo-nazis obtiennent $7,5 \%$ des voix et une représentation parlementaire. En GrandeBretagne, le National Front obtient entre $5 \%$ et $7 \%$ des voix bien que la Dame de fer lui ait enlevé quasiment tous ses thèmes électoraux.

La bête immonde croît, fait des petits, influence les partis politiques traditionnels, pour la plus grande gloire de la race, de la nation et de la propriété privée. Elle enfonce son clou, devient légitime, bouleverse le champ politique et provoque des réalignements vers la droite. Utilisant le prétexte du Front national, le Parti socialiste s'ouvre à une droite (barriste) qui a, elle-même, courtisé Le Pen. Quelle est la solidité de «l'alibi démocratique» censé justifier aux yeux du "peuple de gauche » cette alliance avec la droite, rebaptisée «centre » pour les besoins de la cause, au prix du renoncement à toute velléité de changement? Et, dans ces conditions, quel type d'alternance représenterait une droite lépénisée, autre élément de l'alternative? N'y a-t-il pas lieu de penser que chaque accord du Parti socialiste avec une partie de la droite, pour créer une majorité élargie, constituera un recul, un renoncement 
qui, affaiblissant la confiance dans la possibilité du changement, ne fera que renforcer l'extrême-droite?

Rappelons que le phénomène Le Pen a fait sa percée à l'automne 1983 (Dreux, Morbihan, élections européennes ${ }^{25}$ ). L'émergence du phénomène a coïncidé avec le moment où la gauche au pouvoir (depuis 1981) a radicalement changé de politique économique et sociale. C'est à partir du jour où le premier ministre socialiste Mauroy a commencé à appliquer une politique d'austérité que l'extrême-droite est devenue la force électorale que l'on connaît. Le Pen disait à ce moment-là en Bretagne : «Maintenant je suis le seul à pouvoir faire rêver les Français » ${ }^{26}$.

Cette gauche appliquant l'austérité de façon plus efficace que la droite (les gens hésitant alors à se mobiliser contre «leur» gouvernement), renonçant aux réformes, adoptant l'idéologie néo-libérale, crée un vide politique que l'extrême-droite réussit à combler. Ce vide s'approfondit et rien n'indique un changement de conjoncture et de politique pour les années à venir.

La barbarie émerge de ce vide de perspectives de changement social, de la désespérance qu'il impulse et du retour aux valeurs bourgeoises traditionnelles qu'il implique : travail, famille, patrie, et ce sur un fond de crise de société et d'idéologies de gauche. Le redoutable art démagogique de l'ambiguïté permet à Le Pen de nager dans les eaux mêlées du néo-libéralisme. Il assume cyniquement l'injustice sociale et le mépris des faibles, prône l'inégalité et la concurrence sauvage, ce faisant il nourrit le racisme et ouvre à la régression sociale. Si la classe dirigeante française se revendique de la compétence technocratique, prétend au pragmatisme (la fin des idéologies), se pare des vertus du «modernisme » et de la société transnationale (l'Europe de 1992), Le Pen, par ailleurs, retourne comme un gant cette soi-disant "non-idélogie» pour étaler son chauvinisme, son racisme, son phallocratisme... À l'impératif

25. C'est aux élections municipales de Dreux en septembre 1983 que le Front national est devenu une force polirique importante électoralement, récoltant $16,72 \%$ des suffrages et devenant incontournable quant à la gestion de cette municipalité par la droite. Sur cette lancée, le Front national obtenait $12,01 \%$ des suffrages à l'élection législative partielle du Morbihan de décembre 1983 pour enfin obtenir, en juin 1984, 10,95\% aux élections pour le Parlement européen et 10 députés.

26. Cité par E. Plenel et A. Rollat dans L'effet Le Pen, op. cit., p. 129. 
carégorique de la compétitivité, il oppose l'obsession crépusculaire du déclin. Et s'il s'affiche comme chef, c'est uniquement au nom de sa capacité à donner une voix à toutes les frustrations : il aurait le courage de «dire tout haut ce que les autres pensent tout bas! » ${ }^{27}$

Idéologiquement, comme pour la classe politique française dans son ensemble, Le Pen possède cette hargne à s'accrocher aux dernières colonies et cette propension à désigner les boucs émissaires. Il s'est repu à ces mangeoires idéologiques, les structurant et les rendant conséquentes, donc extrémistes. Il renoue avec les vieilles habitudes bourgeoises refoulées, les révise et leur donne une vitalité nouvelle. Cette brutalité est efficace. Et rien ne nous permet de croire que le monstre ne reviendra pas hanter l'humanité, la piétiner, car elle est en «miettes », et peu s'en faut pour que la croissance de cette peste brune ne se poursuive.

Laissons donc Le Pen nous avertir lui-même du danger. C'est à Compiègne, le 3 mars 1984, qu'il se présente comme suit : "Je suis la bête immonde qui monte... ${ }^{28}$. Pas d'erreur, la menace est là et bien consciente d'elle-même.

28. Cité par S. Dumont, J. Lorieu et K. Criton, op. cit., page de garde. 